Europhys. Lett., 69 (5), pp. 819-825 (2005)

DOI: $10.1209 / \mathrm{epl} / \mathrm{i} 2004-10409-9$

\title{
Experimental studies of front propagation and mode-locking in an advection-reaction-diffusion system
}

\author{
M. S. Paoletti and T. H. Solomon $\left(^{*}\right)$ \\ Department of Physics, Bucknell University - Lewisburg, PA 17837, USA
}

received 22 October 2004; accepted in final form 10 January 2005

published online 9 February 2005

PACS. 82.40. $\mathrm{Ck}$ - Pattern formation in reactions with diffusion, flow and heat transfer.

PACS. 47.70.Fw - Chemically reactive flows.

PACS. $47.52 .+j-$ Chaos.

\begin{abstract}
Experiments are presented on reaction processes in a cellular, time-periodic fluid flow composed of a chain of oscillating vortices. Previous studies have shown that mixing of passive impurities in this flow is chaotic. Chemical fronts are studied in this flow using the excitable regime of the ruthenium-catalyzed Belousov-Zhabotinsky reaction. The velocities of the fronts are measured as a function of both the frequency and the amplitude of the external forcing. Arnol'd tongues are observed, where the propagating front mode-locks to the forcing, resulting in regimes where the front propagates an integer number of vortices in an integer number of drive periods. Overlapping of the tongues results in dual-locked behavior where the propagation velocity switches between two locked values.
\end{abstract}

Numerous dynamical processes are characterized by the propagation of a front across the system. Examples of front propagation can be found in a wide range of physical, chemical and biological systems including solidification [1], combustion [2], ecological [3] and epidemiological [4] processes. The dynamics of these fronts are governed by the interplay between mixing in the system and the interaction between the different species. Several previous studies have examined this phenomenon in the reaction-diffusion limit in which fluid flows are not present and mixing is achieved solely via molecular diffusion [5]. But fluid systems typically are not stagnant; rather, flows in the system dramatically alter and enhance transport and mixing. Despite this fact, the more general advection-reaction-diffusion problem has only recently received attention [6-9], and there have been almost no experimental studies [10-12]. The importance of mixing is particularly significant in light of recent studies indicating that mixing can be chaotic, even for well-ordered, laminar fluid flows [13,14].

In this letter, we present experimental studies of the propagation of chemical fronts in a cellular flow consisting of a chain of oscillating vortices $[15,16]$. In particular, we investigate the propagation velocities for the fronts. We compare the experimental results to a theory [17] that predicts locking of the fronts to the external forcing. Mode-locking of this nature has been found in a variety of physical systems [18,19]; this, however, is the first experimental evidence of mode-locking in an advection-reaction-diffusion system.

(*) E-mail: tsolomon@bucknell.edu

(C) EDP Sciences 
(a)

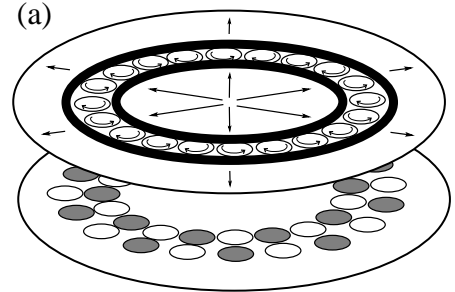

(b) Plexiglass side-walls Electrodes

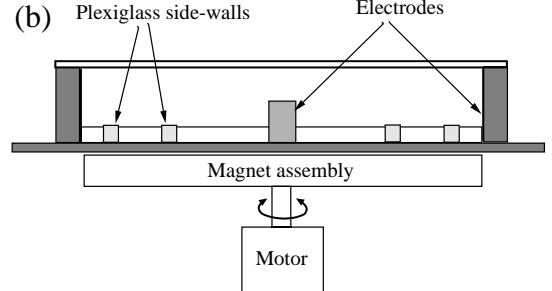

Fig. 1 - Diagram of the flow and experimental apparatus. (a) Exploded view of the magnetohydrodynamic forcing technique. The flow is an annular vortex chain bounded by two plexiglass rings with radii 6.1 and $8.3 \mathrm{~cm}$. (b) Side view of the apparatus. A circular container holds the fluid and the rings. Below this container is the magnet assembly, which is mounted coaxially with a motor.

The flow is shown in fig. 1(a). The vortex chain is confined to an annulus between two bounding rings (drawn in bold). The vortex chain has a wavelength $\lambda=4.5 \mathrm{~cm}$ (two vortex widths). The chain oscillates azimuthally with an angular frequency $\omega$ and amplitude $B$. The oscillation amplitude can be expressed non-dimensionally as a fraction of a vortex width $b \equiv k B / \pi$, where $k=2 \pi / \lambda$. The oscillation frequency is scaled by the advective time $\tau_{\text {adv }} \equiv 2 \pi / k U$ (where $U$ is the maximum flow velocity), resulting in a non-dimensional frequency $\nu=\omega / k U$.

Long-range transport for the stationary $(b=0)$ regime has been shown to be diffusive $[15,16,20]$. For the oscillatory regime $(b \neq 0)$, chaotic advection $[15,16]$ dominates; the resulting transport has also been found $[21,22]$ to be diffusive, except for oscillation frequencies near resonant values where transient superdiffusion is found.

Recently, reaction fronts have been investigated theoretically in this flow $[8,9,17]$. For the oscillatory regime, locking bands are predicted where the front propagates an integer number $N$ of wavelengths in an integer number $M$ of drive periods. When locked, the theory predicts well-defined front velocities:

$$
v_{f}=\frac{N \lambda}{M T}=\frac{N \omega}{M k} .
$$

We define a dimensionless propagation velocity $\xi \equiv v_{f} / U$; the theory therefore predicts

$$
\xi_{\text {locked }}=\frac{N}{M} \nu
$$

The locked propagation velocity is independent of $b$ and the reaction-diffusion (no flow) propagation velocity.

In the experiments presented here, the flow is composed of 20 alternating vortices (10 wavelengths) wrapped into a circle. The flow is forced with a magnetohydrodynamic technique [16, 23], as shown in fig. 1. An electrical current passes radially through a $2 \mathrm{~mm}$ thick layer of fluid and interacts with a spatially varying magnetic field produced by magnets below the fluid. The magnet assembly is mounted coaxially on a motor. A stationary vortex pattern is achieved when the motor is off. Oscillations in the vortex chain are achieved by oscillating the magnet assembly in the azimuthal direction. The experimental flow differs from the model flow of refs. $[15,17,21,22]$ in two respects: a) there are no-slip rather than free-slip boundary conditions at the bottom and sides of the annulus; and b) there is a weak, secondary, three-dimensional flow that slowly pumps fluid inward along the bottom and up through the vortex centers [23].

The chemical reaction used in these experiments is the ruthenium-catalyzed BelousovZhabotinsky system [24-26]. Bromomalonic acid is produced using the method described 


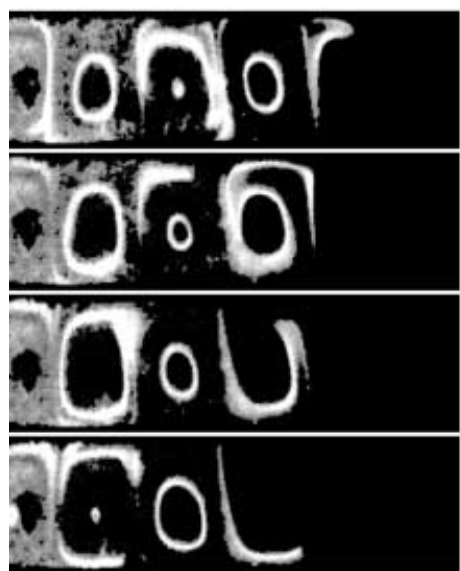

(a)

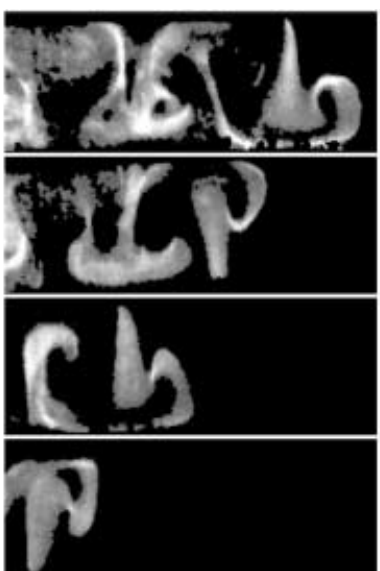

(b)

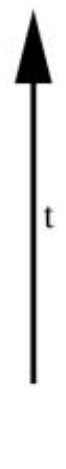

Fig. 2 - Sequences showing front propagation in the oscillating vortex chain. (a) Time-independent flow; images are separated in time by $32 \mathrm{~s}$. (b) Oscillatory flow, $b=0.69, \nu=0.54$; images are separated in time by one drive period $(80 \mathrm{~s})$.

in ref. [26]. To remove inhibitory $\mathrm{Cl}^{-}$ions, $\mathrm{AgNO}_{3}$ [27] is added dropwise to a solution of $\left[\mathrm{Ru}(\text { bpy })_{3}\right] \mathrm{Cl}_{2}$ and the precipitate is filtered. To remove any excess $\mathrm{Ag}^{+}$ions, $\mathrm{NaBr}$ is added dropwise and the precipitate is filtered. Initial concentrations of the reactants for each run are: $[\mathrm{BrMA}]=0.09 \mathrm{M},[\mathrm{MA}]=0.03 \mathrm{M},\left[\mathrm{BrO}_{3}^{-}\right]=0.16 \mathrm{M},\left[\mathrm{H}_{2} \mathrm{SO}_{4}\right]=0.25 \mathrm{M},\left[\left(\mathrm{Ru}(\mathrm{bpy})_{3}\right)^{2+}\right]=$ $2.7 \mathrm{mM}$. With these concentrations, the reaction is excitable and photosensitive. The solution is initially in the $\mathrm{Ru}^{2+}$ state, which is stable and orange in color. A reaction pulse is triggered by placing a thin $(0.25 \mathrm{~mm})$ silver wire into the solution for approximately ten seconds. The silver wire oxidizes the $\mathrm{Ru}^{2+}$ to the $\mathrm{Ru}^{3+}$ state, which is green and absorbs light strongly at $675.2 \mathrm{~nm}$. The $\mathrm{Ru}^{3+}$ propagates as a pulse, oxidizing the $\mathrm{Ru}^{2+}$. Since the $\mathrm{Ru}^{3+}$ propagates as a pulse, the region behind the pulse returns to the $\mathrm{Ru}^{2+}$ state and is able to be re-triggered.

A high-power video projector $(205 \mathrm{~W})$ is used to image an illumination pattern onto the apparatus. Since the reaction is inhibited by blue and white light, everything outside the annular region is illuminated with white light to prevent the initiation of spurious fronts that can invade the region of interest. The region of interest (in the annulus) is illuminated with red light, which does not inhibit the reaction. (Use of red light also improves image contrast between the green $\mathrm{Ru}^{3+}$ and the orange $\mathrm{Ru}^{2+}$ states.) A small section (1-2 vortices wide) of the annulus is illuminated with bright white light, and the reaction front is triggered in the adjacent vortex. The chemical pulse cannot propagate through the brightly illuminated region due to photoinhibition; consequently, the pulse propagates in only one direction, allowing the use of 18-19 vortices in the experiments. Images of the propagating chemical patterns are acquired with a high-resolution, 12-bit CCD camera with a red interference $(676 \mathrm{~nm})$ filter. All of the images shown in this paper are "de-curled" versions of the originals, displayed as though the vortex chain was linear.

For a stationary vortex chain, a front advances in three stages (fig. 2a): a) when initiated within a vortex near a fixed point at one corner, the front is predominately advected around the outside of the vortex to the opposite corner; b) once near the opposite fixed point, the front can advance chemically past the separatrix and trigger a reaction in the next vortex; c) the front, having advected around the outside of the vortex, propagates inward to the 


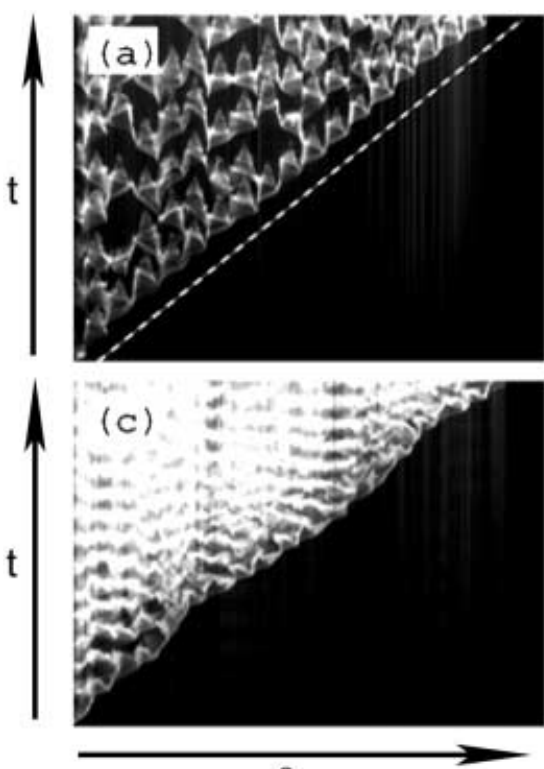

$\theta$
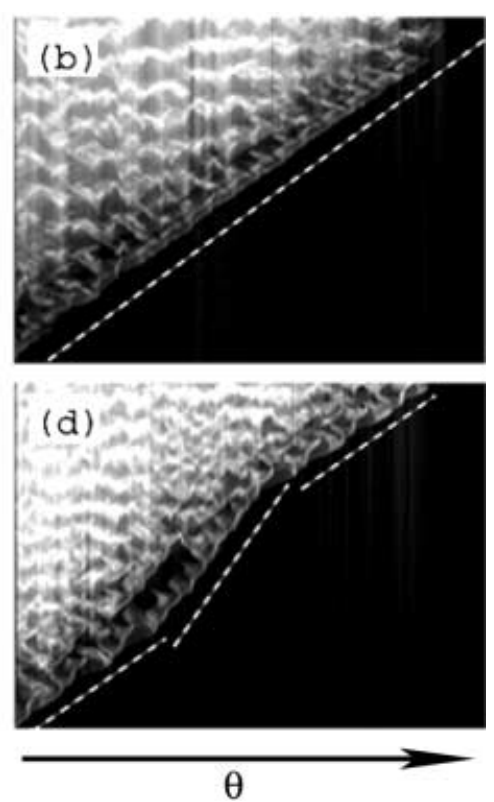

Fig. 3 - Spacetime plots for propagation fronts, obtained by stacking radially summed and decurled images. (a) Time-independent $(b=0)$ flow. (b) Oscillatory flow $(b=0.69, \nu=0.54)$; front is locked to the external forcing. (c) Oscillatory flow $(b=0.52, \nu=0.27)$; front is unlocked. (d) Oscillatory flow $(b=0.69, \nu=0.31)$; front switches between two different locked velocities. The dashed lines show regimes of constant front velocity.

vortex center. The behavior is similar to numerical results observed for the stationary case in ref. [17]. A spacetime plot corresponding to fig. 2a is shown in fig. 3a. The propagation velocity is the inverse of the slope of this plot.

The behavior can be quite different in the oscillatory regime (figs. $2 \mathrm{~b}$ and $3 \mathrm{~b}$ ). Qualitatively, chaotic advection results in stretching and folding of the advancing chemical pulse near separatrices between vortices. (Compare the images in fig. $2 b$ with those in ref. [16].) In some circumstances, this behavior results in the pulse being triggered more quickly in the following vortex, resulting in enhancement in the overall propagation velocity over that for the stationary flow. The opposite effect is also possible - the chaotic mixing might pull the front away from the separatrix, inhibiting triggering of the front in the next vortex and reducing the propagation velocity.

Mode-locking can be seen in the sequence shown in fig. $2 \mathrm{~b}$. The pattern at the front repeats after 2 periods, but is shifted by 2 vortex widths ( 1 wavelength of the flow), corresponding to a locking ratio $(N, M)=(1,2)$.

Other kinds of front propagation behavior are found for the oscillatory flow. Depending on the amplitude and frequency of oscillation, the front might be unlocked, as seen in fig. 3c. For unlocked cases, the propagation velocity itself may not be constant. There are also "duallocked" regimes where the front velocity switches between two different short-term values, as shown in fig. $3 \mathrm{~d}$.

The data for the propagation velocities are summarized in figs. 4 and 5. Figure 4 shows the front velocities as a function of oscillation frequency for a large oscillation amplitude $(b=0.69)$. (For the dual-locked regimes, both front velocities are plotted for the same frequency.) The 


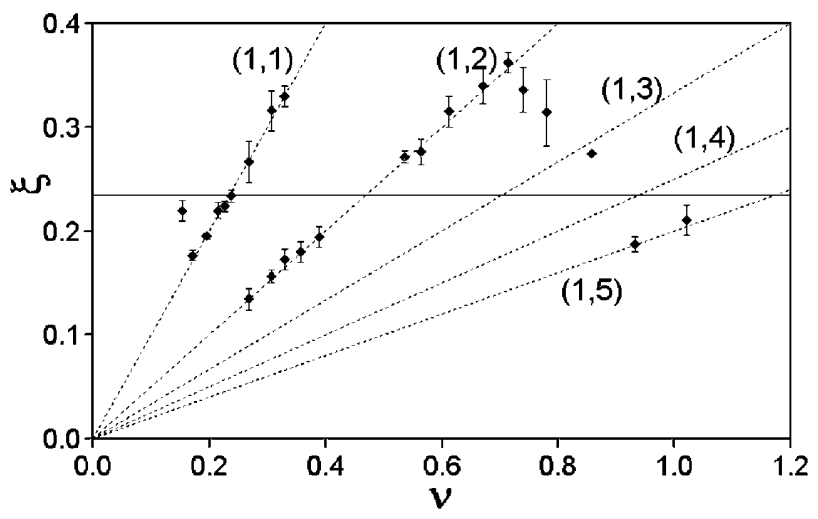

Fig. 4 - Front propagation velocities for oscillatory forcing; $b=0.69$. The velocity for the stationary case (see figs. $2 \mathrm{a}$ and $3 \mathrm{a}$ ) is denoted by the solid horizontal line. The dashed, diagonal lines correspond to the predictions from eq. (2) for mode-locked fronts with locking integers $(N, M)$.

dashed lines plotted on this graph are the predictions for the locked velocities from eq. (2); note that there are no fitted parameters in these predictions. Three locked regimes are evident in this figure corresponding to $(N, M)=(1,1),(1,2)$ and $(1,5)$.

There is overlap between the $(1,1)$ and $(1,2)$ locking bands. In fact, the switching behavior shown in fig. $3 \mathrm{~d}$ corresponds to a run obtained in this overlap region. Previous studies [28] have indicated that chaotic time dependence might be possible in overlapping locking regimes. Longer data runs are necessary to determine the time dependence of the switching to assess whether or not it is chaotic, but this is technically difficult due to the fact that there are only 20 vortices.

Experimentally, we have mapped out the Arnol'd (locking) tongues for the $(1,1)$ and $(1,2)$ mode-locked regimes, as shown in fig. 5. Several different types of behavior are shown in this

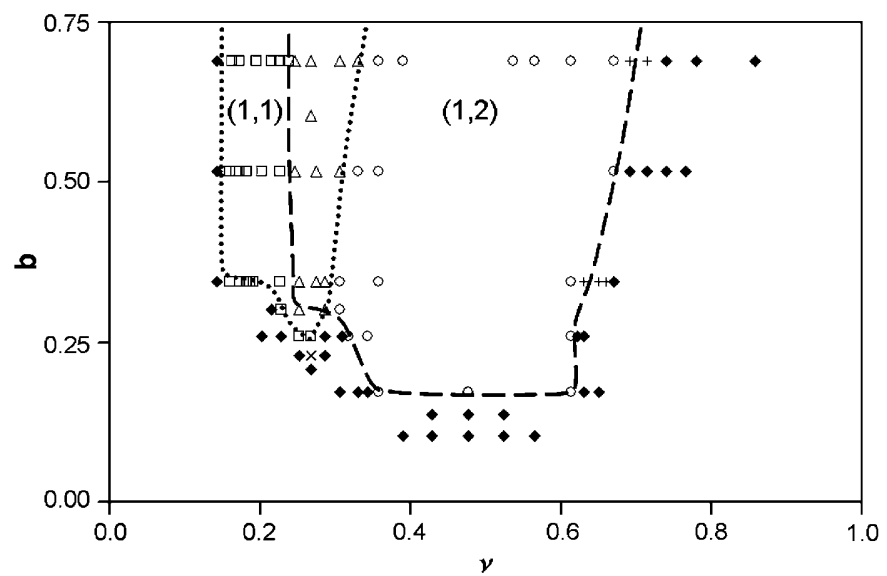

Fig. 5 - Arnol'd tongues for the mode-locked regimes; open squares and circles correspond to locking with $(N, M)=(1,1)$ and $(1,2)$, respectively; open triangles corresponds to dual-locked states; + and $\times$ denotes partially-locked runs; and filled diamond corresponds to unlocked fronts. The dotted and dashed curves show roughly the boundaries of the $(1,1)$ and $(1,2)$ tongues, respectively. 
graph. The most straightforward are the fully unlocked and fully locked cases. But locking in this system is not a simple yes/no proposition. Near the edges of the tongue, the front may be "partially locked". A partially locked front is characterized by a propagation velocity that agrees with the prediction of eq. (2) during $25-75 \%$ of the total run. The dual-locked cases (such as the one shown in fig. 3d) are also shown in fig. 5. These denote the regimes where the $(1,2)$ locking tongue overlaps with the $(1,1)$ locking tongue.

The experimental results shown here agree very well with a recent theoretical prediction for locking of front propagation velocities in the oscillating vortex chain [17]. Considering the differences in the details between the simplified model and the flow from the experiments, the agreement indicates that the locking phenomenon is quite robust and depends more on the overall structure of the flow, rather than the details.

The Arnol'd tongues seen in these experiments are similar in many respects to modelocking observed in other systems, but the locking mechanism is quite different. In previous studies of locking, the systems have well-defined, global, natural frequencies of oscillation. It is these internal frequencies that lock to the frequency of the imposed external forcing. In the advection-reaction-diffusion experiments presented here, the closest thing to a natural, internal frequency would be a typical circulation frequency for a fluid element around a vortex. But the circulation frequency depends on the location within the vortex; for instance, the circulation time diverges for fluid elements that pass near the hyperbolic fixed points. And the flow itself remains unchanged by the mode-locking. It is not the circulation frequency that locks to the external forcing; rather, it is the behavior of the spreading reaction within the flow that somehow locks. It is also interesting that mode-locking is observed in the advectionreaction-diffusion system, even though mixing in this flow is chaotic. From these perspectives, the detailed mechanism is quite different from that for previous studies of mode-locking, and certainly merits additional theoretical discussion.

$$
* * *
$$

We would like to thank A. CATLlá for suggesting that we consider the photosensitive Belousov-Zhabotinsky system for our studies and K. MARTinez for technical advice on the chemistry. These experiments were supported by the National Science Foundation, grants DMR-0071771, DMR-0404961 and REU-0097424.

\section{REFERENCES}

[1] Hurle D. T. J. (Editor), Handbook of Crystal Growth, Vol. 1B (North-Holland, Amsterdam) 1993.

[2] Kupervasser O., Olami Z. and Procaccia I., Phys. Rev. E, 59 (1999) 2587.

[3] Petrovskit S. V., Morozov A. Y. and Venturino E., Ecol. Lett., 5 (2002) 345.

[4] Kuperman M. N. and Wio H. S., Physica A, 272 (1999) 206.

[5] Grindrod P., The Theory and Applications of Reaction-Diffusion Equations: Patterns and Waves (Clarendon Press, Oxford) 1996.

[6] Neufeld Z., Kiss I. Z., Zhou C. S. and Kurths J., Phys. Rev. Lett., 91 (2003) 084101.

[7] Scheuring I., Karolyi G., Pentek T. T. A. and Toroczkai Z., Freshwater Biol., 45 (2000) 123.

[8] Abel M., Celani A., Vergni D. and Vulpiani A., Phys. Rev. E, 64 (2001) 046307.

[9] Abel M., Cencini M., Vergni D. and Vulpiani A., Chaos, 12 (2002) 481.

[10] Ronney P. D., Haslam B. D. and Rhys N. O., Phys. Rev. Lett., 74 (1995) 3804.

[11] Leconte M., Martin J., Rakotomalala N. and Salin D., Phys. Rev. Lett., 90 (2003) 128302. 
[12] Nugent C. R., Quarles W. M. and Solomon T. H., Phys. Rev. Lett., 93 (2004) 218301.

[13] Aref H., J. Fluid Mech., 143 (1984) 1.

[14] Ottino J. M., The Kinematics of Mixing: Stretching, Chaos and Transport (Cambridge University Press, Cambridge) 1989.

[15] Solomon T. H. and Gollub J. P., Phys. Rev. A, 38 (1988) 6280.

[16] Solomon T. H., Tomas S. and Warner J. L., Phys. Rev. Lett., 77 (1996) 2682.

[17] Cencini M., Torcini A., Vergni D. and Vulpiani A., Phys. Fluids, 15 (2003) 679.

[18] Olemskoi A. I. and Klepikov V. F., Phys. Rep., 338 (2000) 571.

[19] Lin A. L., Hagberg A., Meron E. and Swinney H. L., Phys. Rev. E, 69 (2004) 066217.

[20] Solomon T. H. and Gollub J. P., Phys. Fluids, 31 (1988) 1372.

[21] Solomon T. H., Lee A. T. and Fogleman M. A., Physica D, 157 (2001) 40.

[22] Castiglione P., Crisanti A., Mazzino A., Vergassola M. and Vulpiani A., J. Phys. A, 31 (1998) 7197.

[23] Solomon T. H. and Mezic I., Nature, 425 (2003) 376.

[24] Scott S. K., Oscillations, Waves and Chaos in Chemical Kinetics (Oxford University Press, Oxford) 1994.

[25] Kuhnert L. and Krug H.-J., J. Phys. Chem., 91 (1987) 730.

[26] Reddy M. K. Ram, Nagy-Ungvarai Zs. and Muller S. C., J. Phys. Chem., 98 (1994) 12255.

[27] For many of the runs - in particular, the data used to map out the $(1,2)$ branch - we used $\mathrm{Ag}_{2} \mathrm{SO}_{4}$ instead of $\mathrm{AgNO}_{3}$.

[28] Jensen M. H., BaK P. and Bohr T., Phys. Rev. A, 30 (1960) 1984. 